\title{
Life satisfaction as the main factor behind the elderly's health knowledge utilization: A qualitative study in an Iranian context
}

\author{
Sima Nedjat ${ }^{1}$, Robab Sahaf*2, Hamid Reza Khankeh ${ }^{3}$, Reza Fadayevatan², Reza Majdzadeh ${ }^{4}$, Masoud Karimlou ${ }^{5}$
}

Received: 27 Sep 2017

Published: 20 Nov 2018

\section{Abstract}

Background: The elderly population's health has become a priority as their numbers are on the rise and they are increasingly becoming vulnerable to physical and mental diseases. Studies show that an elderly person's health depends on his/her utilization of health knowledge in daily life. Hence, here we investigated the contributing factors of knowledge utilization in the elderly population.

Methods: A qualitative study was conducted through conventional content analysis. Semi-structured in-depth interviews were held with 29 elderly individuals from Tehran. A focus group discussion was conducted (eight elderly individuals), and an expert panel was held with nine experts to complement the results.

Results: Upon data analysis, four categories and seven subcategories were extracted. The main categories included provision of basic needs, maintaining dignity, life satisfaction, and negative feelings toward self and others. The concept of 'life satisfaction' held a pivotal role in relation to the other categories.

Conclusion: Life satisfaction was the main category in the utilization of health knowledge among the elderly. Aging should be foreseen and forethought to increase life satisfaction. The followings can be effective in increasing life satisfaction in the elderly: Promoting positive-thinking, placing greater emphasis on spiritualism in life, employment of the elderly, and promoting the culture and tradition of respecting the elderly.

Keywords: Knowledge utilization, Life satisfaction, Old people, Elderly, Iran

Conflicts of Interest: None declared

Funding: None

\section{*This work has been published underCC BY-NC-SA 1.0 license.}

Copyright $₫$ Iran University of Medical Sciences

Cite this article as: Nedjat S, Sahaf R, Khankeh HR, Fadayevatan R, Majdzadeh R, Karimlou M. Life satisfaction as the main factor behind the elderly`s health knowledge utilization: A qualitative study in an Iranian context. Med J Islam Repub Iran. 2018 (20 Nov);32:115. https://doi.org/10.14196/mjiri.32.115

\section{Introduction}

The average age of the world population has been on the rise. From the beginning of the 19th century to the end of the 20th century life expectancy increased from 47 to 78 years in the United States of America, and exceeded 80 years in Japan (1). Also, In Iran, life expectancy has gone from a little above 50 years in 1977 to above 70 years in the recent years (2). Iran is a developing country, which is going through a demographic transition, wherein the elderly

Corresponding author: Dr Robab Sahaf, robabsahaf@gmail.com

1. Department of Aging, University of Social Welfare and Rehabilitation, Knowledge Utilization Research Center, Tehran University of Medical sciences, Tehran, Iran

2. Iranian Research Center on Aging, Department of Aging, University of Social Welfare and Rehabilitation Sciences, Tehran, Iran

3. Department of Health in Emergency and Disaster and Nursing, University of Social Welfare and Rehabilitation, Tehran, Iran, Department of Clinical Science and Education, Karolinska institute, Stockholm, Sweden

4. Department of Epidemiology and Biostatistics, School of Public Health and Knowledge Utilization Research Center, Tehran University of Medical Sciences, Tehran, Iran

5. Department of Epidemiology and Biostatistics, Faculty of Tehran Medical Sciences, Islamic Azad University, Tehran, Iran population is growing in number. The elderly's perspective is traditional, and does not match the new generation's mechanical life. Phenomena like the elderly nursing home is a taboo in their opinion, and $\mathrm{v}$ alue conflict is on the rise (3, 4). The aging of the population has been associated with an increased significance of elderly health. The health of this group has a significant impact on their quality of life as well

$\uparrow$ What is "already known" in this topic:

In 2010 a clinical trial found interventions that increased life satisfaction could promote health among the Japanese elderly.

\section{$\rightarrow$ What this article adds:}

Life satisfaction is associated with social, economic and familial factors and improving the lives of the elderly and promoting their QOL can raise life satisfaction among them. Promoting a positive thinking morale, strengthening spirituality in life, employment and promotion of the culture and tradition of respecting the elderly can be influential in raising life satisfaction in the elderly. 
as on that of other groups (5). Although aging is a physiological process and does not directly lead to disease, it is accompanied by various problems that may include physiological, mental and social aspects as a result of reduced vital capacities (6). Most elderly people are affected by chronic and debilitating diseases, and experience the physiological changes of aging as well (7). Therefore, in addition to promoting the extent of life through improvement of healthy aging, it is also important to focus on promoting its quality.

In the recent years, the increase in the number and quality of research has led to the production of considerable volumes of knowledge in all sciences, including healthy aging. Based on the available literature, a wide gap exists between knowledge and its utilization (8). This temporal gap may have direct consequences for the elderly population in particular. In other words, the health of the elderly population may improve with the utilization of scientific evidence in healthy aging (9). In this particular field, we can expect to observe improvements in longevity and health following the utilization of health knowledge. What is certain is that the backbone of health knowledge utilization is the acquisition of health knowledge in the first place. Many studies have been conducted on the significance of promoting health literacy and its impact on one's health (10). However, more than few cases have been observed in the elderly wherein health knowledge has been acquired, but not utilized. In other words, health literacy has been achieved, but no change has been created in the individual's lifestyle. The Ministry of Health and Medical Education of Iran report on elderly health in 2013 did not give an acceptable account of their health in Iran, and it appears that even those who have acquired health knowledge show no interest in utilizing it (11).

Lee et al. did not observe a significant association between health knowledge and its application (12). Hence, it seems that many prerequisites need to be met in addition to knowledge acquisition to culminate in change (9). Therefore, health literacy alone will not guarantee its application.

Here we aimed to investigate the contributing factors or reasons related to health knowledge utilization in the Iranian elderly. We sought to find why the elderly do not apply the acquired health knowledge to practice, and why they do not change their lifestyle (13). This study may be justified by the following points: The positive impact of utilizing health knowledge in the elderly's quality of life, the significance of its contributing factors, and the fact that no such study has been conducted in Iran to date (to our knowledge). An insight into the Iranian elderly's experience may help us design effective interventions to promote quality of life (QOL) among the elderly as well as achieving longevity.

\section{Methods}

The reason and manner with which the Iranian elderly utilize health knowledge is a subjective, complex, multifactorial and context-bound phenomenon. Therefore, we chose the qualitative method of research to reach a deeper understanding of this phenomenon. The research team held mul- tiple sessions, with the participants selected through purposeful sampling and maximum variation for age, literacy and health status. To this end, participants ranged from illiterate to highly educated, debilitated and ill to active and healthy, and included both men and women. However, it should be noted that in a qualitative study, the samples are not representative. Inclusion criteria were as follows: Age> 60 years, resident of Tehran, and adequate level of consciousness and willingness to participate in the interview. The location and timing of the interviews were set according to the participants' preferences. Overall, 29 (15 women and 14 men) in-depth interviews, a focus group discussion (eight elderly individuals) and an expert panel (nine experts) were conducted. The participants' age ranged from 60 to 86 years. The interviews lasted for 20 to 45 minutes, and the duration depended on the participant's level of cooperation and interest. However, the FGDs and expert panels were 75 and 90 minutes long, respectively.

The research team comprised of six individuals: Two gerontologists, a physician, a qualitative methodologist, and two experts in the field of knowledge translation and utilization. The interviews were conducted by a researcher, who had attended qualitative study workshops and courses, and had conducted three interviews in the presence of the team's methodologist and one of the gerontologists. The participants' data were collected through in-depth interviews with no prior background of the research question. The interview guide was developed. The interviews began by asking the participants about their healthy behaviors and life style compared to their health knowledge. Then, the facilitators and barriers for health knowledge utilization were inquired, followed by questions on the ways in which it was acquired, and the circumstances under which it would or would not be utilized. All the participants were Persian speaking, and there was no difficulty in this regard. Field notes were taken in some of the interviews and used as supplementary data for trustworthiness.

In this study, we applied the qualitative content analysis method and analyzed the data, using constant comparative analysis (recommended by Strauss and Corbin) (14). Data collection and analysis were performed simultaneously. The analysis consisted of many stages; first, the recorded interviews were transcribed after being listened to two to three times. After the open coding stage, the extracted codes were checked with the research team. Then, the codes and meaning units relevant to the research question were tabulated. The following concepts were registered against each code after repeated reading and rereading, such that some meaning units were assigned concepts up to five stages. However, the following concepts were more abstract compared to the preliminary concepts. After reaching saturation, to achieve greater certainty, we conducted the last three interviews and the focus group discussion (FGD) with eight elderly individuals. After extracting the categories and subcategories, an expert's panel consisting of nine experts was held. The experts included three gerontologists, two qualitative methodologists, two epidemiologists, one academic member and one researcher in the psychological and social sciences domain. Experts' opinion was recorded 
and transcribed and consensus was reached. Guba \& Lincoln's strategies were applied to ensure trustworthiness of data; the four criteria of credibility, dependency, conformability and transferability were observed (15). The credibility of data collection was ascertained by triangulation; i.e., in addition to conducting semi-structured interviews, field notes were also taken, and prolonged engagement was also applied. Dependency was assured by peer and member check. Peer check was done through multiple sessions with the research team. In addition to checking the members' impressions after the code extraction, a FGD was held with the participants and a number of new individuals to ensure member check. Conformability was assured by taking into account the other researchers' opinions, and transferability was guaranteed by extensively describing all the stages of the task. The research proposal was approved by the Ethics Committee of University of Social Welfare and Rehabilitation Sciences (USWR). Before the interviews and audiorecordings and after sufficiently explaining the project to the elderly, informed consent was taken from all of them. The participants were assured that their identities would remain confidential and that their voices would not be aired anywhere. Some cases insisted that part or all of their voices not be recorded; in those cases, the interviews were recorded through note taking alone. Furthermore, member withdrawal was possible at any stage of the study. The participants were given gifts of appreciation at the end of the interviews.

\section{Results}

Overall, four main categories or concepts were extracted, the summary of which has been presented in Table 1 . The main categories we as follows: Provision of basic needs, maintaining dignity, life satisfaction and negative feelings toward self and others.

\section{Provision of Basic Needs}

Based on the participants' experiences, the provision of basic needs is a prerequisite and sometimes vital in the lives of human beings, especially for older adults. Two subcategories were extracted from this category: Physical- economic needs, and emotional- communication needs. Some of the participants believed that putting health knowledge to practice was beyond expectation when life's basic needs were not provided. For example:

"The more basic a need the stronger it is... When someone can only sleep five hours a day to make ends meet exercising becomes a luxury." (p8, 62 year-old male)

\section{1- Provision of Physical-Economic Needs}

Most participants believed that they should have certain facilities if they were to apply what they knew. Economic issues play an influential role here. A 68 year-old retired teacher (p10) said:

"Pensioners have low incomes and high expenditures. We have grandchildren; how long can we keep telling them we do not have money? So, we are forced to cut from our daily expenses. We no longer care for quality; the cheaper is better. Then you visit a doctor and s/he tells you to take this and that; I have to pay from my pocket, so I can't."
1.2- Provision of Emotional - Communication Needs

This was the other subcategory extracted. Based on the participants' opinions, emotional - mental needs are provided through love-seeking, noticing an individual's values, being viewed gracefully, prominence, peace and tranquil, care, emotional and verbal communication, and social and family ties. Having emotional and verbal communication was very important to the elderly; all the participants addressed its significance. According to one participant:

"An elderly has communication and humanistic needs. This is the most important nourishment, because she feels she's finished, she's no good, she's gone, nobody wants her, and she is a burden ... Physical nourishment comes after spiritual nourishment.... Once she's got energy ...her nutrition will become fine, she'll re-gain her appetite." (p1, 73 year-old female)

\section{1- Maintaining Dignity}

People usually attain their dignity in the society by the role they adopt in life. Maintenance of dignity has a great impact on people's lives. This category comprised three subcategories: Employment, role change, and marginalization.

\section{1- Employment}

Employment has a positive impact on maintaining one's dignity. Some participants even believed their viability depended on it. Once they lose their job, the elderly feel as if they have lost their dignity and it is unbearable for most of them.

"When I retired I said to myself "I've got to work"; work is what keeps me alive...." (p1, 73 year-old female)

2.2- Changing Roles

Participants believed that as they age, their capabilities decline in some fields and that they are no longer able to assume their roles as before. They have difficulty facing the fact that they have lost the roles that they once assumed their whole lives. Hence, it would be better to assign them new roles; this way their valuable experience can be used and at the same time they would not feel as if they were being marginalized. Another participant said:

"I feel I will definitely grow old sooner if I am rejected. If I withdraw myself I'll have a feeling of emptiness, unless I have a substitute role." (p2, 63 year-old male)

\section{3- Marginalization}

Being marginalized creates a difficult situation for the elderly. Rejection, loss of purpose, transition, and the lack of a need to change were all influential in this subcategory. The participants believed that the progress of technology has accelerated the marginalization of the older generation. One participant commented as follows:

"Don't tell her not to touch it if she wants to try it out; explain it to her... this is how it works. At one time when we were young and very talented, we learned everything, but now the novel technology is alien to us." (p18, 63 year-old female)

\section{2- Life Satisfaction}

Most participants believed satisfaction from life and its relevant factors to be influential in putting health knowledge to practice. When there is life satisfaction, the 
Table 1. Categories and subcategories and examples of the contributing factors of utilizing health knowledge

\begin{tabular}{|c|c|c|c|}
\hline Category & Subcategory & Some of the Codes & Meaning Units \\
\hline \multirow[t]{3}{*}{$\begin{array}{l}\text { 1- Provision of basic } \\
\text { needs }\end{array}$} & $\begin{array}{l}\text { 1.1Physical - } \\
\text { economic needs }\end{array}$ & Appropriate nutrition & $\begin{array}{l}\text { "Pensioners have low incomes and high expenditures. We } \\
\text { have grandchildren; how long can we keep telling them we } \\
\text { do not have money? So, we are forced to cut from our daily } \\
\text { expenses. We no longer care for quality; the cheaper the bet- } \\
\text { ter. Then you visit a doctor and s/he tells you to take this and } \\
\text { that; I have to pay from my pocket, but I cannot afford it." (p } \\
\text { 10) }\end{array}$ \\
\hline & $\begin{array}{l}1.2 \text { Emotional - com- } \\
\text { munication needs }\end{array}$ & Being with family & $\begin{array}{l}\text { "In whatever condition we are, we should be residing in our } \\
\text { own home and with our families... one of the things that af- } \\
\text { fect me is my relationship with my daughter; for example, } \\
\text { when she comes at night she caresses me." (p 1) }\end{array}$ \\
\hline & & Verbal communication & $\begin{array}{l}\text { "We prefer verbal communications... all the elderly like to } \\
\text { be spoken to." ( } \mathrm{p} 18)\end{array}$ \\
\hline \multirow[t]{4}{*}{$\begin{array}{l}\text { 2- Maintaining dig- } \\
\text { nity }\end{array}$} & 2.1 Employment & $\begin{array}{c}\text { Being employed and active in } \\
\text { old age }\end{array}$ & $\begin{array}{l}\text { "When I retired, I said to myself, "I've got to work"; work is } \\
\text { what keeps me alive." ( }(\mathrm{p} 1)\end{array}$ \\
\hline & 2.2 Changing roles & Substitute roles & $\begin{array}{l}\text { "I'll work even if I have to work for free; I won't sit idly; I } \\
\text { have goals." (p 12) }\end{array}$ \\
\hline & 2.3 Marginalization & Rejection & $\begin{array}{l}\text { "I feel I will definitely grow old sooner if I am rejected. If I } \\
\text { withdraw myself, I will have a feeling of emptiness, unless I } \\
\text { have a substitute role ... aging does not make one old and } \\
\text { weary, loss of purpose does." (p 2) }\end{array}$ \\
\hline & & Transition & $\begin{array}{l}\text { "We had no difficulty with the technology of our own time, } \\
\text { but the countless developments in new technology have su- } \\
\text { perseded us." ( }(\mathrm{p} 25)\end{array}$ \\
\hline \multirow[t]{3}{*}{ 3- Life satisfaction } & 3.1 Internal factors & Feeling useful & $\begin{array}{l}\text { "It is important to know that you are still seen by others and } \\
\text { that you are still helpful to others." ( }(\mathrm{p} 29)\end{array}$ \\
\hline & 3.2 External factors & $\begin{array}{l}\text { Reception of attention from } \\
\text { spouse and others nearby }\end{array}$ & $\begin{array}{l}\text { "It will boost your morale when they pay attention to what } \\
\text { you say and do, it doesn't matter even if they don't do it, but } \\
\text { when they listen, you know that you are still important, and } \\
\text { that they accept you." (p 29) }\end{array}$ \\
\hline & & Being respected & $\begin{array}{l}\text { "When she called me 'mother' I didn't mind; but if she'd } \\
\text { called me 'an old woman' I'd have told her not to call me } \\
\text { that again." ( }(\mathrm{p} \mathrm{18)}\end{array}$ \\
\hline \multirow[t]{3}{*}{$\begin{array}{l}\text { 4- Negative feelings } \\
\text { toward self and others }\end{array}$} & & Being humiliated by others & $\begin{array}{l}\text { "Disliking oneself is a kind of stubbornness itself; sometimes } \\
\text { we want to retaliate against others... we have been humili- } \\
\text { ated so much." ( } \mathrm{p} 20)\end{array}$ \\
\hline & & Loneliness & $\begin{array}{l}\text { "Loneliness and being ignored are the worst things that can } \\
\text { happen to an elderly." ( } \mathrm{p} 1)\end{array}$ \\
\hline & & Feeling like a burden & $\begin{array}{l}\text { "I do not want to be a burden on anyone; my daughter has a } \\
\text { husband and kids... I do not even want to bother my own } \\
\text { daughter." }(\mathrm{p} 25)\end{array}$ \\
\hline
\end{tabular}

individual cares more about his/her health, and applies the health knowledge that he/she has acquired. According to one participant:

"When you're satisfied with your life, you care more about your health... you do what is good for your health." (p5, 76 year-old female)

Life satisfaction consists of two intertwined subcategories of internal and external factors. These two greatly influence each other.

\section{1- Internal Factors Affecting Life Satisfaction}

The internal factors affecting life satisfaction were as follows: Spirituality and participation in religious gatherings, visiting sick people, helping others, positive thinking, and self-liking. One participant exclaimed:

"I try to help others; for example, when I visit a sick elderly person like myself, whose above 60 years, I feel more peaceful. At the same time, I have made that person happy too. In other words, the good deed you do comes back to you. When you do such things and go to bed at night you thank God a million times for being able to do what you did." (p17, 69 year-old female)
Positive thinking and overlooking the negative aspects of life was also considered influential on life satisfaction.

"You have to be positive about life; you shouldn't look for the negative all the time." (p25, 60 year-old female)

3.2- External Factors Affecting Life Satisfaction

The following fell into this subcategory: Being agreeable and respected by others, happiness and joy, and having a feeling of security.

The participants believed that agreeableness is achieved when the followings are fulfilled: Receiving attention from one's spouse and those close to you, feeling useful, selfliking, positive thinking and reception of adequate support. In this regard, one participant said:

"It's important to be agreeable. It will boost your morale when they pay attention to what you say and do, because you will know that you are still important, and that they accept you. It is important to know that you are still seen by others and that you are still beneficial to others..." (p29, 78 year-old male)

The participants believed that every moment of joy brings life satisfaction. 
"Satisfaction is important. I find it strange when someone commits suicide... Why did that person do such a thing? Every moment of this life is filled with joy... I always pray to God to prolong my life so I can enjoy it more..." ( $(\mathrm{p} 2,63$ year-old)

One participant considered joy and happiness to be dependent on the feeling of security:

"... When you feel secure in every aspect of your life (social, welfare and other aspects), one automatically pursues happiness. Otherwise, we are constantly trying to stock to our deficits; we have no time to be happy." (p20, 75 yearold female)

\section{3- Negative Feelings toward Self and Others}

Negative feelings toward self and others are developed following feelings of humiliation, loneliness, being overlooked, and feeling like a burden. These feelings culminate in disliking oneself and others; in retaliation, the elderly exhibits stubbornness and neglect their health. When they are humiliated and feel that they are no longer loved, they develop feelings of negative self-perception, and retaliate by being stubborn and careless toward their own health. One participant said:

"Disliking oneself is a kind of stubbornness itself; sometimes we want to retaliate against others... We have been humiliated so much that we no longer think about ourselves." (p20, 75 year-old female)

Some participants attributed their negative feelings toward self and others and dissatisfaction with life to their loneliness and lack of communications:

"Loneliness and being ignored are the worst things that can happen to an elderly... In whatever condition we are, we should be residing in our own home and with our families... Preferably, the family members should always be with us; if not, then they should visit us as much as possible." (p1, 73 year-old female)

\section{Discussion}

Here we investigated the contributing factors of health knowledge utilization in the life of the Iranian elderly. The participants' experiences provided us with four concepts: Provision of basic needs, maintaining dignity, life satisfaction, and negative feelings toward self and others. However, as the main category, the concept of 'life satisfaction' had a fundamental relationship with the other categories. Therefore, the 'life satisfaction' category will be deliberated first and then the remaining three categories and their associations with the main category will be discussed.

1. Life Satisfaction: The participants addressed the significance of life satisfaction in utilizing health knowledge, both explicitly and implicitly. They believed that they would care about their health and put their knowledge to practice when and if they were satisfied with their lives. Life satisfaction was the main factor in adopting healthy behavior, and in other words utilizing health knowledge. In this study, provision of basic needs, maintaining dignity and negative self-perception were closely associated with life satisfaction. Review of the literature revealed that life satisfaction brings more health to people. Niknami et al. conducted a descriptive correlational study on 320 members of Rasht's Pensioners' Club, and found a positive correlation between life satisfaction and healthy behaviors (16). According to another study, a significant association was found between life satisfaction and health knowledge utilization (17). Yamadi et al.'s clinical trial also found that those interventions that increased life satisfaction could promote health among the Japanese elderly (18). Gow et al. believe that among the various contributing factors, life satisfaction has the greatest impact on successful aging (19).

Life satisfaction is the feeling one has toward his/her life as a whole. Life satisfaction is influenced by circumstances as well as the individual's impression and outlook towards them. An individual's life and the goals that he has set for himself and the extent to which those goals are achieved determine a person's life satisfaction (20). Life satisfaction and needs are interrelated; hence, as needs change so does the former. On the other hand, if one's goals are not set according to one's true needs, dissatisfaction is bound to ensue. According to Wirtz et al., the determining factors of life satisfaction varied across different cultures and nations (21).

In this study, agreeableness, spirituality and happiness were associated with life satisfaction. Agreeableness is achieved through respect, positive thinking, and reception of adequate support. Chang et al. linked positive thinking to life satisfaction and negative thinking to depression (22). Feeling supported helps people cope with difficulties and shortcomings. Bagheri-nesami et al. conducted a study on the home-residing elderly in the city of Sari, and found a direct association between life satisfaction and social support (23). Those who are respected by others and are considered respectable and productive individuals have higher levels of life satisfaction $(24,25)$.

2. Provision of Basic Needs: Most of the participants believed that provision of basic needs was a prerequisite for health knowledge utilization. As long as one's basic needs have not been met, caring for one's health is beyond expectation. Provision of basic needs has a direct effect on an individual's life satisfaction. Abraham Maslow has depicted human needs in a hierarchical manner where the other needs cannot be addressed unless the basic ones are first met. In his opinion, physical needs fall into the category of primary needs and precede emotional, mental and security needs (26). However, most participants in this study believed that emotional, human and social communications were stronger than physical needs and were of greater priority. Certain studies confirm this finding. According to Liu et al.'s study greater satisfaction is achieved by living with family, as human and social communications and interactions are greater (27). Overall, close and pleasant interactions with friends and family increase life satisfaction in the elderly (28).

3. Maintaining Dignity: Old age is a time when an elderly's experiences can be flourished and utilized. The elderly should not imagine that they are no longer capable of doing things. They should not be stripped of their roles all at once. If a role is taken from them, it should be replaced with another that suits their capabilities and morale. Since aging is associated with a reduction in one's performance 
and capabilities, and at the same time one's appearance changes, life satisfaction can greatly influence adaptation to new circumstances and roles. Wang et al. have associated the elderly's marginalization and distancing with depression and dissatisfaction with life (29).

This study found that transition has caused a greater gap between the generations and has marginalized the elderly. The staggering growth of technology is one reason why the elderly are constantly left behind. Although some middleaged and elderly individuals are trying to keep pace with technological developments, the overt speed of innovations has made the older generations surrender.

Where maintenance of dignity is concerned, the literature review points toward the significance of employment and involvement in life satisfaction of the elderly. Clark et al.'s study showed the positive and significant effect of employment in an elderly's life satisfaction, QOL and health (30). Yamada claimed that one of the few non-aggressive interventions that can actually influence life satisfaction in the elderly is employment (18).

4. Negative Feelings toward Self \& Others: Here, the factors leading to dissatisfaction and development of negative feelings toward self and others were as follows: Humiliation by others, loneliness and disrespect, which sometimes even culminate into a retributive attitude, stubbornness, self-disliking and non-utilization of health knowledge.

Life satisfaction is a complex concept, through which one can assess his sense of being. It can bring about zest and enthusiasm, lack of indifference, accountability, endurance in the face of difficulties, sense of purpose and enthusiasm to achieve it, acceptance of one's appearance and self, adaptation to new circumstances, and positive thinking and morale (31). Life satisfaction indicates a positive inference of the outside world. A positive outlook toward life and life satisfaction are associated with self-confidence and pride. In addition, life satisfaction itself is affected by personal and cultural factors, employment, lifestyle, wisdom and spirituality, emotional and love relationships (32-35). Life satisfaction is assessed by comparing an individual's needs and his assets and resources. It is achieved when one's assets conform to one's needs. Naturally, when an individual's assets and resources are less than his needs, the person becomes dissatisfied with his life.

Strengths and Weaknesses of the Study: To our knowledge, this study was the first of its kind to investigate the contributing factors of health knowledge utilization in an elderly Iranian population. Semi-structured interviews were conducted to draw a clear picture of the topic and to better understand health knowledge utilization in the Iranian elderly. When conducting a qualitative study, we do not attempt to generalize the findings. Nevertheless, since the 'health knowledge utilization' concept is dependent on cultural, environmental, and ethnic variations across Iran, we recommend conducting similar studies in different cultures and different elderly groups, such as the lonely elderly, the vulnerable elderly and the chronically-ill elderly, using grounded theory.

\section{Conclusion}

When people are generally satisfied with their lives, they care about their health and utilize their health knowledge. Life satisfaction is associated with social, economic and familial factors. Therefore, improving the lives of the elderly and promoting their QOL can raise life satisfaction among them. A positive outlook toward life and its achievements are also influential in this regard. Hence, whatever strengthens this positive outlook becomes important; spirituality and religious behavior also gain significance. Therefore, different programs should be designed in advance and aging should be anticipated to improve the status of health knowledge utilization and life satisfaction among the elderly. Based on our findings, promoting a positive thinking morale, strengthening spirituality in life, employment and promotion of the culture and tradition of respecting the elderly can be influential in raising life satisfaction in the elderly. Conducting further studies to identify the key factors, affecting life satisfaction in the elderly is highly recommended.

\section{Conflict of Interests}

The authors declare that they have no competing interests.

\section{References}

1.The world health report 2003: shaping the future. World Health Organization. 2003.

2. Poor Malek F, Abolhassani F, Naghavi M, Mohammad K, Majdzadeh $\mathrm{R}$, Holakouie Naeini K, et al. Direct estimation of life expectancy for population of Iran in year 2003. Arak Med Uni J. 2007;10(1):18-30.

3. Ahmadi V, Ghasemi V, Kazemipour S. Role of demographic transition in sociological changes of the family. Women Stud J. 2012;10(1).

4. Momeni K, Karimi H. Comparison of mental health between elderly admitted in sanitarium with elderly in sited in personal home. J Kermanshah Univ Med Sci. 2011;14(4).

5. Worrall G, Knight J. Our elderly population feels well. They are in control and not burdening their caregivers. Canad Fam Physic 2004;50:1662

6. Halter JB. Hazzard's geriatric medicine and gerontology. McGraw-Hill Companies. 2009.

7. Monahan F. Phipps' Medical-Surgical Nursing Health \& Illness Perspectives. Missouri: Elsevier Mosby. 2007:1428-1429.

8. Lomas J. The in-between world of knowledge brokering. BMJ. 2007; 334(7585):129-132.

9. Lavis JN, Lomas J, Hamid M, Sewankambo NK. Assessing countrylevel efforts to link research to action. Bullet World Health Organiz. 2006;84(8):620-628.

10. Health COSDO. Closing the gap in a generation: health equity through action on the social determinants of health: final report of the commission on social determinants of health. 2008.

11. Motlagh M, Taheri Tanjani P. Elderly health profile in Islamic Republic of IRAN. Minist Health Med Edu. 2014.

12. Lee SYD, Tsai TI, Tsai YW, Kuo KN. Health literacy, health status, and healthcare utilization of Taiwanese adults: results from a national survey. BMC Pub Health. 2010;10(1):614.

13. Sadat Madah S, Emami A, Foroughan M, Rahgozar M, Norouzi K, Mohammadi F. The status of social and leisure time activities in the elderly rising in Iran and Sweden. Salmand. 2008;8(3):597-606.

14. Graneheim UH, Lundman B. Qualitative content analysis in nursing research: concepts, procedures and measures to achieve trustworthiness. Nurs Edu Today. 2004;24(2):105-112.

15. Schwandt TA, Lincoln YS, Guba EG. Judging interpretations: But is it rigorous? Trustworthiness and authenticity in naturalistic evaluation. New Direct Evalu. 2007;114:11-25.

16. Niknamy M, Namjoo A, Baghaee M, Atrkar Roshan Z. Survey the Relationship between Life Satisfaction and Health Behaviors in Elderly People Referring to Active Retire mental Centers. J Guilan Uni Med Sci. 2010;19:46-54.

17. Sung MS, Song BS. Health promoting behavior and degree of life satisfaction in rural elderly people. J Kore Acad Fund Nurs. 
2004:11(1):82-91.

18. Yamada T, Kawamata H, Kobayashi N, Kielhofner G, Taylor RR. A randomised clinical trial of a wellness programme for healthy older people. Br J Occup Ther. 2010;73(11):540-548.

19. Gow AJ, Pattie A, Whiteman MC, Walley LJ, Deary IJ. Social support and successful aging: Investigating the relationships between lifetime cognitive change and life satisfaction. J Indiv Dif. 2007:28(3): 103-115.

20. Headey B. Life goals matter to happiness: A revision of set-point theory. Social Indic Res. 2008;86(2):213-231.

21. Wirtz D, Chiu CY, Diener E, Oishi S. What Constitutes a Good Life? Cultural Differences in the Role of Positive and Negative Affect in Subjective Well-Being. J Person. 2009;77(4):1167-1196.

22. Chang EC, Sanna LJ. Optimism, pessimism, and positive and negative affectivity in middle-aged adults: a test of a cognitiveaffective model of psychological adjustment. Psychol Aging. 2001;16(3):524.

23. Bagheri-Nesami M, Sohrabi M, Ebrahimi MJ, Heidari-Fard J, Yanj J, Golchinmehr S. The Relationship between Life Satisfaction with Social Support and Self-efficacy in Community-dwelling Elderly in Sari, Iran, 2012. J Mazandaran Univ Med Sci. 2013;23(101):38-47.

24. Georgellis Y, Tsitsianis N, Yin YP. Personal values as mitigating factors in the link between income and life satisfaction: Evidence from the European Social Survey. Socia Indic Res. 2009; 91(3):329-344.

25. Zullig KJ, Ward RM, Horn T. The association between perceived spirituality, religiosity, and life satisfaction: The mediating role of selfrated health. Soc Indic Res. 2006;79(2):255-274.

26. McLeod S. Maslow's hierarchy of needs. Simply Psychol. 2007;1.

27. Liu LJ, Guo Q. Loneliness and health-related quality of life for the empty nest elderly in the rural area of a mountainous county in China. Quali Life Res. 2007;16(8):1275-1280.

28. Heinrich LM, Gullone E. The clinical significance of loneliness: A literature review. Clinic Psychol Rev. 2006;26(6):695-718.

29. Wang JJ, Snyder M, Kaas M. Stress, loneliness, and depression in Taiwanese rural community-dwelling elders. Int $\mathrm{J}$ Nurs Stud. 2001;38(3):339-347.

30. Clark F, Jackson J, Carlson M, Chou CP, Cherry BJ, Jordan-Marsh $\mathrm{M}$, et al. Effectiveness of a lifestyle intervention in promoting the wellbeing of independently living older people: Results of the Well Elderly 2 Randomised Controlled Trial. J Epidemiol Commun Health. 2012;66(9):782-790.

31. Diener E, Inglehart R, Tay L. Theory and validity of life satisfaction scales. Social Indic Res. 2013;112(3):497-527.

32. Masoudi R, Soleimani MA, Qorbani M, Hasheminia AM, Pour Dehkordi AH, Bahrami N. The effect of family centered empowerment model on the quality of life in elderly people. J Qazvin Uni Med Sci. 2010;14(1):57-64.

33. Serrano JP, Latorre JM, Gatz M, Montanes J. Life review therapy using autobiographical retrieval practice for older adults with depressive symptomatology. Psycho Age. 2004;19(2):272.

34. Heravi Karimloo M, AnooshehM, Foroughan M, Taghi Sheykhi M, Hajizade E, Sadat M, et al. Loneliness from the perspectives of elderly people: a phenomenological study. Salmand Iran J Age. 2008;2(4): 410-420.

35. Kudo H, zumo Y, Kodama H, Watanabe M, Hatakeyama R, Fukuoka $\mathrm{Y}$, et al. Life satisfaction in older people. Geriatr Geronto Int. 2007;7(1):15-20. 